\title{
Recognition of Fissures in Bony Structures through Image Processing
}

\author{
Holman Montiel $^{\# 1}$, Edwar Jacinto ${ }^{\# 2}$, Fredy Martinez ${ }^{\# 3}$ \\ \#Universidad Distrital Francisco José de Caldas, Facultad Tecnológica \\ Cll 68 D Bis A Sur No. 49F - 70, Bogotá D.C., Colombia \\ ${ }^{1}$ hmontiela@udistrital.edu.co, ${ }^{2}$ ejacintog@udistrital.edu.co \\ ${ }^{3}$ fhmartinezs@udistrital.edu.co
}

\begin{abstract}
This document shows the design of a software for the detection of very pronounced edges, fissures and fractures of bone structures in radiological images, using image processing techniques as an enhancement method. A graphic interface was used for the user to select the bone to inspect, by means of an object labeling algorithm, the coordinates of said bone, "region of interest" ROI are identified, after which the thresholding of the image will be performed, then a series of morphological operations will be applied and in this way the detection of the lesion, which is highlighted by the change of color for the visualization in the output image, making an overlap of the fissures and / or fractures in the original image.
\end{abstract}

Keywords - Image processing, Biomedical computing, Fissures recognition

\section{INTRODUCTION}

The use of medical image processing as a help for medical diagnosis, presents a number of applications [1], [2], some of which have a determined ROI (region of interest) [3], [4], while others try to get to determine this region using different segmentation techniques [5], some of these techniques use characteristic extraction by iterative search of certain structures [6], some others seek certain type of fissures, cracks or ruptures in structures to analyze [3], others look through statistical methods [7] regions or characteristics to analyze. At the same time, behavioral analysis of certain organs, muscle and bone structures, dental [8] can be performed using non-invasive methods [9], taking the information from electrodes, resonances and digitalized radiographs.

In this application the user determines the ROI by means of the mouse cursor on the digitalized radiography, after this a series of Cartesian validations are carried out to determine exactly which area of the image will be analyzed looking for the vortices of the same [10], after having the segmented image, a series of morphological operations is carried out to determine fissures or fractures [3], [2] on the selected bone, at the end an enhancement process is carried out so that the user can identify and analyze the lesion on the bone to study.

This analysis is currently carried out for images of 2D digitalized radiographs, but in a future work this same methodology can be applied for 3D diagnostic images [8], [11], in order to analyze in more detail, the nature of the damage on the bone.

The analysis of the characteristics, structures and formations of the human body can not only be used for diagnostic tasks and detection of diseases, pathologies or injuries, in addition to this applications could be used to identify people [12], chek of characteristics of medicine alternative that verify the state of certain parts and organs of the body, such as the analysis of the eye and tongue [13], skull bones [14] or another specific part of the human anatomy that can yield information to analyze.

To understand the methods of detecting fissures or breaks on the bone, an explanation of the image operations used to perform said task and how the extraction of the ROI to be analyzed is performed.

\section{METHODOLOGY}

\section{A. Opening and Closing Function}

It is based on the algebra of sets as a form of reduction or equalization of vertices based on a structuring object, the writing based on sets for the function opening is given by the letter A that identifies the set and the structuring object $\mathrm{B}$ :

$$
\mathrm{A} \circ \mathrm{B}=(\mathrm{A} \ominus \mathrm{B}) \oplus \mathrm{B}
$$

For the case of the closing function the set $\mathrm{A}$ is treated with the structuring object $\mathrm{B}$ of the form:

$$
\mathrm{A} \cdot \mathrm{B}=(\mathrm{A} \oplus \mathrm{B}) \ominus \mathrm{B}
$$

It is the following example it can be observed the effect of a spherical structuring object with radius $\mathrm{R}$ that is applied to an object with several dimensions. 

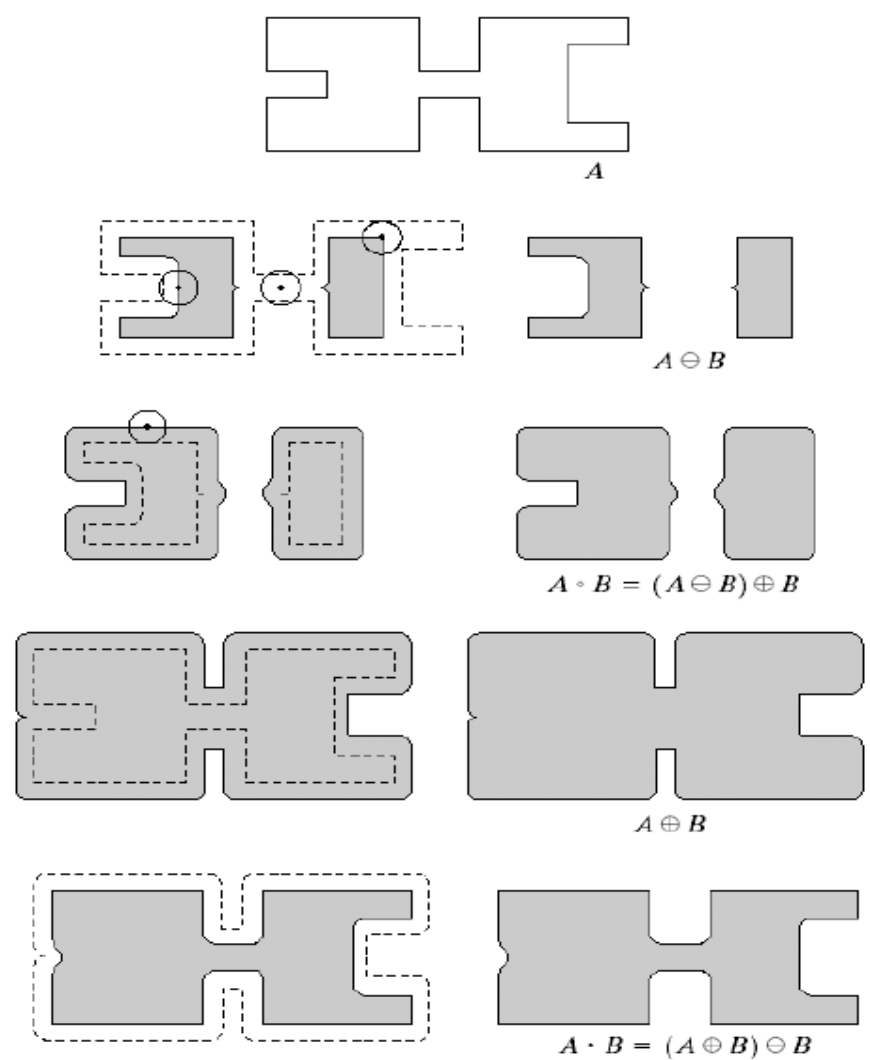

Fig. 1. Application of the Opening-Closing function [15]

It can be seen that based on the structuring object with radius $\mathrm{R}$ the object to be treated is traversed step by step, in the areas where the structuring object B occupies a larger space between the areas of the object, it removes them as seen in the second line of images, followed by the expansion of the object A based on the structuring object B an expansion of the object is obtained and therefore a larger area to perform the opening and closing. At the end of the whole assembly process it is reduced the region of the figure that had a contact greater than the value of the diameter of the structuring object and the rest of the regions of part A are conserved.

\section{B. Bwlabel Function}

The bwlabel function with the syntax for use in matlad described as [L, num] = bwlabel (BW, n) analyzes a black and white image (BW) in which it assigns a numbering to an element of the image, this assigns a number 0 or 1,1 for totally white areas and 0 for totally black areas. After this the value $n$ is assigned so that the function connects 4 or 8 nearby objects with the value in the matrix of 1 as necessary, in the case of omitting this default value would be 8 nearby objects.

The matrix $L$ has as elements the values as element 0 which are the black background, 1 for the first detected element, 2 for the second detected element and so on for the identification of the $\mathrm{N}$ objects that appear in the image for them. It will be seen in Fig. 2 an example of the assignment. 


$$
\begin{aligned}
B W & =\left[\begin{array}{llllllll}
1 & 1 & 1 & 0 & 0 & 0 & 0 & 0 \\
1 & 1 & 1 & 0 & 1 & 1 & 0 & 0 \\
1 & 1 & 1 & 0 & 1 & 1 & 0 & 0 \\
1 & 1 & 1 & 0 & 0 & 0 & 1 & 0 \\
1 & 1 & 1 & 0 & 0 & 0 & 1 & 0 \\
1 & 1 & 1 & 0 & 0 & 0 & 1 & 0 \\
1 & 1 & 1 & 0 & 0 & 0 & 1 & 0 \\
1 & 1 & 1 & 0 & 0 & 0 & 0 & 0
\end{array}\right] \\
B W & =\left[\begin{array}{lllllllll}
1 & 1 & 1 & 0 & 0 & 0 & 0 & 0 \\
1 & 1 & 1 & 0 & 2 & 2 & 0 & 0 \\
1 & 1 & 1 & 0 & 2 & 2 & 0 & 0 \\
1 & 1 & 1 & 0 & 0 & 0 & 3 & 0 \\
1 & 1 & 1 & 0 & 0 & 0 & 3 & 0 \\
1 & 1 & 1 & 0 & 0 & 0 & 3 & 0 \\
1 & 1 & 1 & 0 & 0 & 0 & 3 & 0 \\
1 & 1 & 1 & 0 & 0 & 0 & 0 & 0
\end{array}\right]
\end{aligned}
$$

Fig. 2. Matrix generated by the bwlabel function

In the Fig. 2 the BW matrix it can be observed how the function assigns the values of 1 to the white regions found and the values 0 for the black regions, in the matrix $L$ after making the connection for values close to 4 or next 8 the function will assign the numbering ascending for each region that it finds on the image and is reflected with the ascending values in the matrix, in this case region 1, 2 and 3 respectively.

\section{IMPLEMENTATION AND RESULTS}

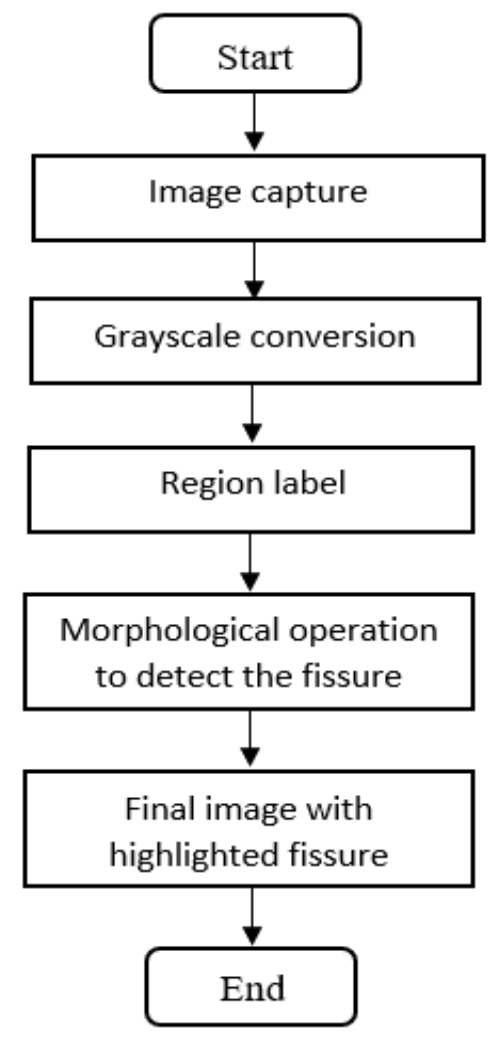

Fig. 3. Flow diagram

The analysis focuses on the identification of bone structure based on mathematical morphology for zones of abrupt change on its structure (for the identification of fractures and bone fissures) based on the flow chart of the Fig. 3, the image is captured and escalated, receives a treatment for conversion to grayscale and later black and white, after the treatment the selection of the region that the user previously made as shown in Fig. 4 will be used and thanks to the function bwlabel previously mentioned will take the area of interest. 
This area will be compared to its shape with reconstructions based on the opening and closing function with a structuring object that eliminates the most acute points, then with the original image and the new smoothed image the difference will be obtained in the form of superposition that in this particular case is the fissure painted red on the original figure. It will be easier to observe the damage on the bone structure for its treatment.

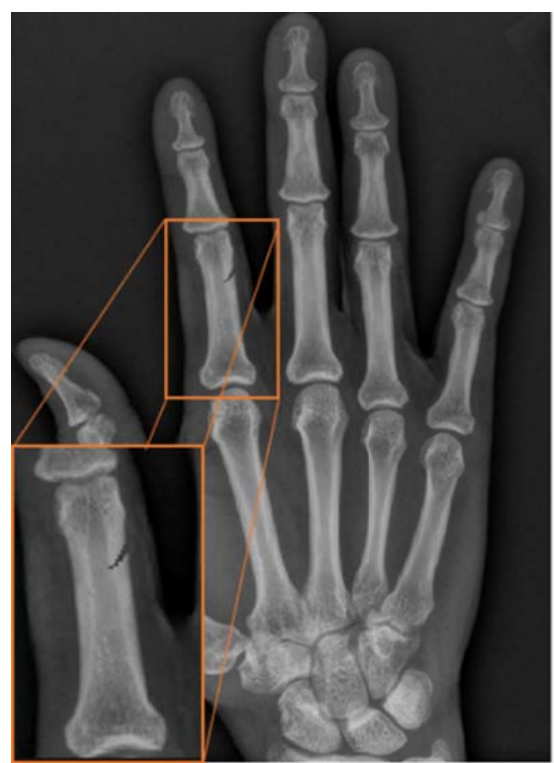

Fig. 4. Radiograph with fissure and increase on it.

The parameters established for this function are given by:

$$
\begin{aligned}
& \text { se1 = strel('disk',5); } \\
& \text { im_obt_close = imclose(im_obt,se1); } \\
& \text { not_im_obt = bitxor(im_obt,1); } \\
& \text { fisura = bitand(im_obt_close,not_im_obt); }
\end{aligned}
$$

Where:

- $\quad$ se1: establishes the structuring object, in this case a disk with radius equal to 5.

- im_obt_close: starts the "closing" and the displacement of the structuring object by the edges of the object to be processed, as shown in the Fig. 5.
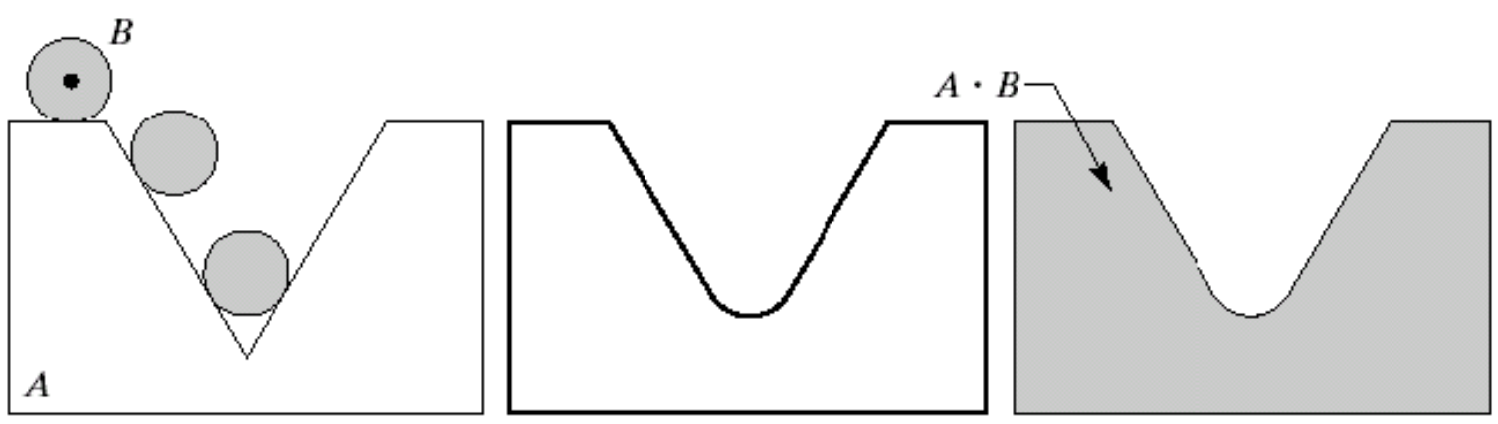

Fig. 5. Process of "closing" based on a structuring object [15]

- not_im_obt: with a window value of color space already established $(v e n=40)$ it negates the image to contrast the fissure in the original figure.

- fissure: it counteracts the two images both the original with the previously negative image to obtain only the fissure and then highlight it based on the original Fig. 4. 
Next, it will be seen the algorithm developed in Matlab:

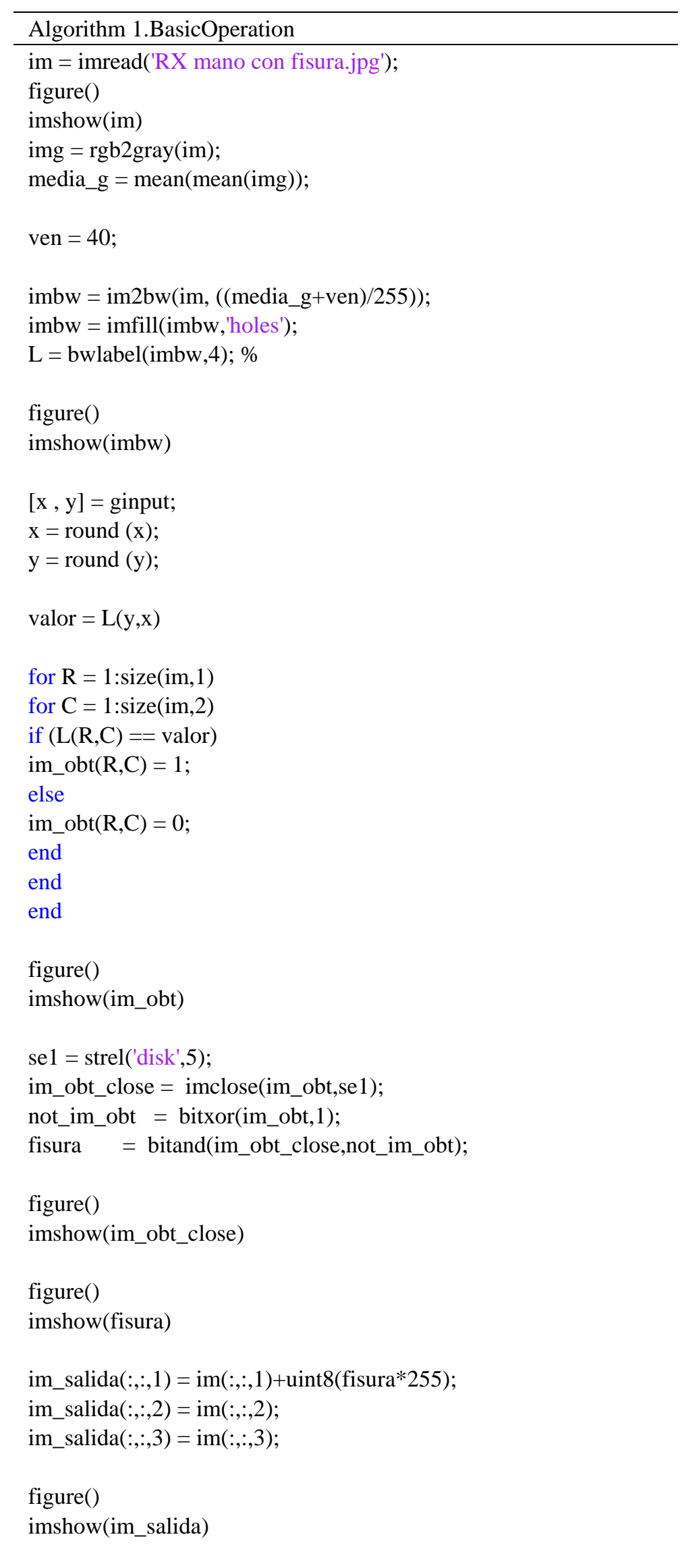


The results obtained will be shown in Fig. 6 where are taken cuts of the figures obtained during the execution of the algorithm in Matlab.

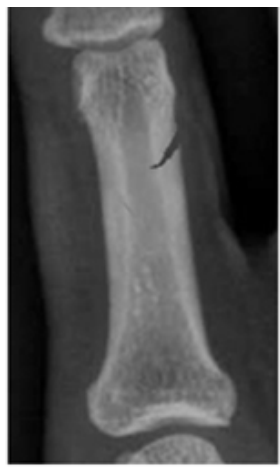

(a)

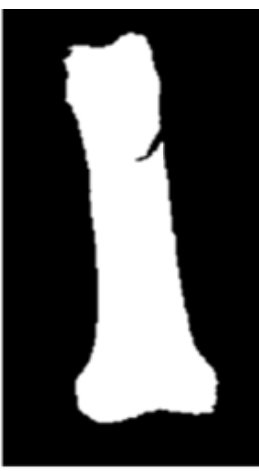

(b)

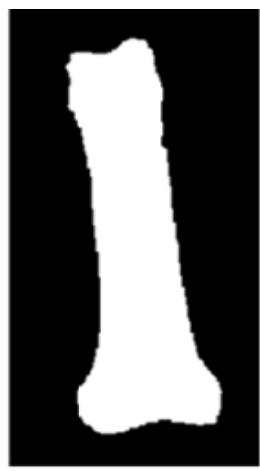

(c)

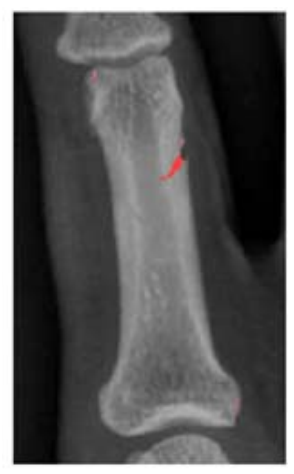

(d)

Fig. 6. Images obtained from the process

The Fig. 6(a) shows the original figure or radiograph enlarged to clearly see the fissure at this scale, Fig. 6(b) is the image treated with grayscale and subsequently converted to black and white, with the morphological operations observed in the Fig. 6(c) the smoothing and post elimination of both fissures and abrupt changes on the figure, finally at the exit of the algorithm it can be seen the fissure on the bony structure highlighted, without an approach it is difficult to recognize without the color highlight.

The algorithm was applied to a series of selected images, with fissures and ruptures, in some hardly differentials, the selection of the region runs by hand of the user that believes to have identified a fissure and this one wants to confirm it with the previously worked algorithm.

The detection of fissure was achieved in 95\% of the radiographs applied as shown in Figure 7, the algorithm was applied to 40 images of different body areas in which one or multiple fissures and breaks were evident, within $5 \%$ resultant, the fissures were highlighted, but so were the elements that appeared in the image as artifacts and very pronounced edges of the x-ray images. The execution time from the taking of the coordinate to the view of the highlighted fissure was 0.77 seconds, tests performed in Windows 10, 64-bit with an Intel Core I7-4720HQ CPU @ 2.60Hz and 8 GBytes of RAM.

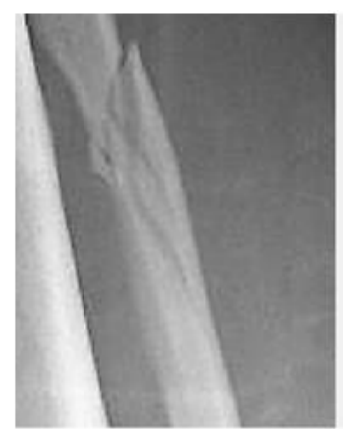

(a)

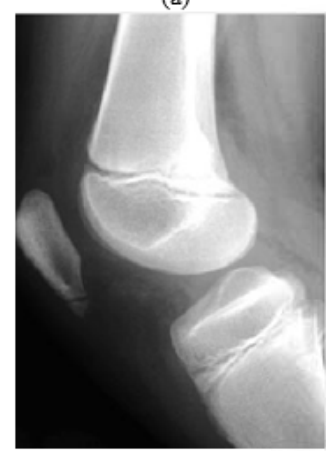

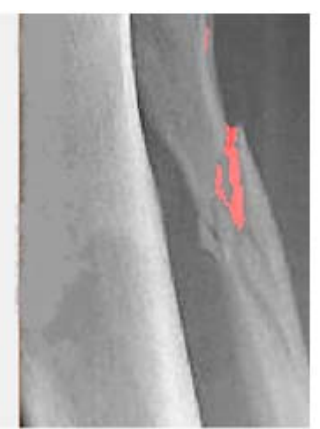

(b)

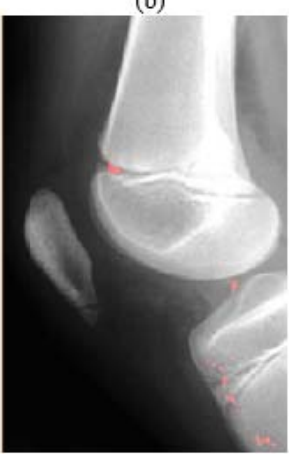

Figure 7. Radiographs with fissure enhancement 


\section{CONCLUSIONS}

A software application that performs the detection of fissures and / or fractures in digitalized radiographs as a diagnostic tool was made, highlighting the region of interest by changing the color of the monochromatic image to a red color, so that the person who is making the diagnosis can verify in detail the bone injury in the analyzed bone.

A series of tests were performed on different diagnostic images with the software implemented, verifying that the lesions were detected in a satisfactory manner, this with the design of a small graphical interface that allows pointing with the mouse pointer the bone to be analyzed and so this way to isolate the algorithmic problem; these tests were satisfactory in $95 \%$, since in some cases the fissure was in edges or artifacts of the radiography, which made it difficult to detect them using the mentioned technique.

On the other hand, the effectiveness of the use of morphological operation in the analysis and detection of edges, fissures or fractures of some types of binary images was verified, within which are the diagnostic images of monochromatic bones.

\section{ACKNOWLEDGMENT}

This work was supported by the Universidad Distrital Francisco José de Caldas Technological Faculty. The views expressed in this paper are not necessarily endorsed by University. The authors thank the research group ARMOS for the evaluation carried out on prototypes of ideas and strategies

\section{REFERENCES}

[1] A. Anand, I. Moon, and B. Javidi, “Automated Disease Identification with 3-D Optical Imaging: A Medical Diagnostic Tool,” Proc. IEEE, vol. 105, no. 5, pp. 924-946, 2017.

[2] C. Xiao, B. C. Stoel, M. E. Bakker, Y. Peng, J. Stolk, and M. Staring, "Pulmonary Fissure Detection in CT Images Using a Derivative of Stick Filter,” IEEE Trans. Med. Imaging, vol. 35, no. 6, pp. 1488-1500, 2016.

[3] B. Lassen, E. M. Van Rikxoort, M. Schmidt, S. Kerkstra, B. Van Ginneken, and J. M. Kuhnigk, "Automatic segmentation of the pulmonary lobes from chest CT scans based on Fissures, Vessels, and Bronchi,” IEEE Trans. Med. Imaging, vol. 32, no. 2, pp. 210 222, 2013.

[4] M. Germino, A. J. Sinusas, C. Liu, and R. E. Carson, "Direct em reconstruction of kinetic parameters from list-mode cardiac PET," 2014 IEEE Nucl. Sci. Symp. Med. Imaging Conf. NSS/MIC 2014, pp. 2-5, 2016.

[5] A. Singh and M. K. Dutta, "A blind \& fragile watermarking scheme for tamper detection of medical images preserving ROI," 2014 Int. Conf. Med. Imaging, m-Health Emerg. Commun. Syst. MedCom 2014, pp. 230-234, 2014.

[6] G. Cao, Y. Liu, and K. Suzuki, “A new method for false-positive reduction in detection of lung nodules in CT images,” Int. Conf. Digit. Signal Process. DSP, vol. 2014-Janua, no. August, pp. 474-479, 2014.

[7] J. Sedlar, M. Bajger, M. Caon, and G. Lee, "Model-Guided Segmentation of Liver in CT and PET-CT Images of Child Patients Based on Statistical Region Merging,” 2016 Int. Conf. Digit. Image Comput. Tech. Appl. DICTA 2016, pp. 1-8, 2016.

[8] M. Lashgari, M. Shahmoradi, H. Rabbani, and M. Swain, "Missing Surface Estimation Based on Modified Tikhonov Regularization: Application for Destructed Dental Tissue,” IEEE Trans. Image Process., vol. 27, no. 5, pp. 2433-2446, 2018.

[9] M. J. M. Cluitmans and P. G. A. Volders, "Influence of body-surface geometry accuracy on noninvasive reconstruction of electrical activation and recovery in electrocardiographic imaging,” Comput. Cardiol. (2010)., vol. 44, pp. 1-4, 2017.

[10] T. C. Hsung, J. Lo, M. M. Chong, T. K. Goto, and L. K. Cheung, "Orbit Segmentation by Surface Reconstruction with Automatic Sliced Vertex Screening,” IEEE Trans. Biomed. Eng., vol. 65, no. 4, pp. 828-838, 2018.

[11] D. Ravichandran, M. G. Ahamad, and M. R. A. Dhivakar, "Performance analysis of three-dimensional medical image compression based on discrete wavelet transform,” Virtual Syst. Multimed. (VSMM), 2016 22nd Int. Conf., pp. 1-8, 2016.

[12] A. Meraoumia, S. Chitroub, and A. Bouridane, "Robust Multispectral Palmprint Identification System by Jointly Using Contourlet Decomposition \& Gabor Filter Response,” pp. 190-197, 2012.

[13] C. M. Huo, H. Zheng, H. Y. Su, Z. L. Sun, Y. J. Cai, and Y. F. Xu, "Tongue shape classification integrating image preprocessing and Convolution Neural Network,” 2017 2nd Asia-Pacific Conf. Intell. Robot Syst. ACIRS 2017, pp. 42-46, 2017.

[14] K.-Y. Chang, Y.-H. Wu, W.-L. Lin, S.-J. Chen, and L.-S. Chen, "Vessel segmentation based on bone-to-bone elimination in brain CT angiography,” 2016 IEEE Int. Conf. Consum. Electron., vol. 1, no. c, pp. 1-2, 2016.

[15] F. Prieto, “Proceso Morfológico en Imágenes Introducción,” pp. 1-84, 2009. 